\title{
ORIENTAÇÕES NUTRICIONAIS PARA A PREVENÇÃO DE ALERGIAS ALIMENTARES EM LACTENTES ${ }^{1}$
}

\author{
NUTRITIONAL GUIDELINES FOR THE \\ PREVENTION OF FOOD ALLERGIES IN INFANTS
}

\author{
Carla Adriane Schneider ${ }^{2}$ e Priscila Berti Zanella ${ }^{3}$
}

\section{RESUMO}

A alergia alimentar é uma doença consequente a uma resposta imunológica que apresenta sintomas que afetam tanto a saúde quanto o estilo de vida dos indivíduos. Suas causas estão possivelmente associadas a fatores genéticos e à exposição ambiental. Dos fatores ambientais a gestação e os primeiros meses de vida tem grande influência tanto na saúde quanto no desenvolvimento das alergias alimentares. Assim, o objetivo deste estudo é revisar as evidências científicas referentes à prevenção de alergias alimentares, analisando os períodos de gestação, amamentação e introdução alimentar. Revisou-se a literatura dos últimos 50 anos, buscando nas bases de dados Medical Literature Analysis e Retrieval System Online, PubMed, e Scientific Electronic Library Online. Os principais consensos e diretrizes aconselham consistentemente todas as mães terem uma dieta balanceada sem restrições durante a gravidez, uma vez que, restringir alérgenos alimentares para mulheres grávidas parece ter pouco ou nenhum efeito sobre a alergia alimentar na primeira infância. Amamentação exclusiva para os primeiros 4 a 6 meses de idade, com introdução alimentar posterior sem adiar a introdução alimentar de alimentos potencialmente alergênicos. Entretanto os estudos são heterogêneos com delineamentos diferentes, durações de amamentação não uniformes e critérios diagnósticos variáveis para alergia alimentar. Desta forma, conclui-se que a restrição de alimentos durante a gravidez, lactação e introdução alimentar apresenta resultados conflitantes em relação a prevenção das alergias alimentares e mesmo que a importância da dieta e sua relação com alergia alimentar não seja contestada mais estudos são necessários para fomentar as recomendações nutricionais.

Palavras-chave: Gravidez; Aleitamento materno; Nutrição da Criança.

\section{ABSTRACT}

Food allergy is a disease resulting from an immune response that presents symptoms that affect both the health and the lifestyle of individuals. Its causes are possibly associated with genetic factors and environmental exposure. From environmental factors, pregnancy and the first months of life have a great influence both on health and on the development of food allergies. Thus, the objective of this study is to review the scientific evidence regarding the prevention of food allergies, analyzing the periods of pregnancy, breastfeeding and food introduction. The literature of the last 50 years was reviewed, searching the Medical Literature Analysis and Retrieval System Online, PubMed, and Scientific Electronic Library Online databases. The main consensus and guidelines consistently advise all mothers to have a balanced diet without restrictions during pregnancy, since restricting food allergens to pregnant women appears to have little or no effect on early childhood food allergy. Exclusive breastfeeding for the first 4 to 6 months of age, with subsequent food introduction without delaying the food introduction of potentially allergenic foods. However, studies are heterogeneous with different designs, non-uniform breastfeeding durations and variable diagnostic criteria for food allergy.

1 Trabalho de Conclusão de Curso de Pós-Graduação em Nutrição em Pediatria - iPGS

2 Nutricionista Especialista em Nutrição em Pediatria

3 Nutricionista Mestre em Ciências Pneumológicas. E-mail: priscila_zanella@hotmail.com 
Thus, it is concluded that the restriction of food during pregnancy, lactation and food introduction presents conflicting results in relation to the prevention of food allergies and even though the importance of the diet and its relationship with food allergy is not contested, further studies are needed to promote nutritional recommendations.

Keywords: Pregnancy; Breast Feeding; Child Nutrition.

\section{INTRODUÇÃO}

A alergia é uma reação específica a uma substância normalmente inofensiva (alérgeno) (VALENTA et al., 2015). Por sua vez, a alergia alimentar pode ser definida como uma doença consequente a uma resposta imunológica anômala, comumente mediada por imunoglobulina E (IgE), que ocorre após a ingestão ou contato com determinado alimento (EIWEGGER et al., 2019). Os alérgenos alimentares são, em sua maioria, glicoproteínas hidrossolúveis resistentes a alterações de temperatura e à ação de acidos e proteases, que por possuírem essas características não sofrem desnaturação e deterioração no trato gratrointestinal podendo ocasionar sua absorção em sua forma inteira (UNTERSMAYR; JENSEN-JAROLIM, 2008). Pessoas com alergias alimentares apresentam sintomas que afetam tanto sua saúde quanto seu estilo de vida, então há um interesse considerável em maneiras de reduzir o risco de desenvolver uma alergia alimentar. As principais manifestações clínicas são reações cutâneas, gastrintestinais, respiratórias até reações sistêmicas como anafilaxia que pode levar ao óbito (CIANFERONI; MURARO, 2012; SCHUSSLER et al., 2017).

Estima-se que as alergias alimentares acometem cerca de $10 \%$ da população (GUPTA et al., 2019) e apresenta nos últimos anos prevalência crescente (TANG; MULLINS, 2017). Os dados mostram ainda que as alergias alimentares afetam mais crianças que adultos (SICHERER; SAMPSON, 2018). Na população infantil os principais alimentos envolvidos nas alergias alimentares são leite de vaca, ovo, amendoim, trigo, soja, peixe, frutos do mar e nozes(MURARO; HERNANDEZ, 2020).

As causas da alergia alimentar possivelmente estão relacionadas a fatores genéticos e à exposição ambiental (BJORKSTÉN, 2005; BENEDÉ et al., 2016; CHANG; WU; LU, 2020). Fatores genéticos são não modificável, portanto, as estratégias para prevenir a alergia alimentar e a maior parte dos estudos científicos tem focado sua atenção na exposição ambiental (SZAJEWSKA, 2012; GOLDSMITH et al., 2016; PERKIN et al., 2016). Sendo a gravidez juntamente com o nascimento até os 24 meses momentos bastante sensíveis durante os quais a alimentação tem uma grande influência no desenvolvimento de saúde (HALFON et al., 2014) e sabendo que esse período pode ter influência no desenvolvimento das alergias alimentares na infância ou até mesmo posteriormente na vida adulta (GAMBONI; ALLEN; NIXON, 2013; FUJIMURA et al., 2019) o objetivo deste estudo é revisar as principais evidências científicas referentes à prevenção de alergias alimentares, analisando os períodos de gestação, amamentação e introdução alimentar. 


\section{METODOLOGIA}

O presente trabalho trata-se de uma revisão criteriosa da literatura a partir de publicações científicas de estudos originais, sem restrição de idioma, dos últimos 50 anos. Foram selecionados artigos indexados nas bases de dados Medical Literature Analysis e Retrieval System Online (MEDLINE), PubMed, e Scientific Electronic Library Online (SciELO), além de sumarizar os pareceres das diretrizes e consensos atuais sobre o assunto. As palavras-chave utilizadas na busca foram: "alergia a alimentos", "hipersensibilidade alimentar", "gestação", "aleitamento materno", "fórmulas infantis" e “alimentação complementar" nos idiomas inglês, português e espanhol.

\section{DESENVOLVIMENTO}

\section{GESTAÇÃO}

Sabe-se que fetos podem ser sensibilizados aos alimentos que suas mães consomem pela exposição transplacentária (CHATZI et al., 2008; KEMP et al., 2011). Vários estudos foram realizados ao longo dos anos para tentar comprovar ou descartar a associação entre o consumo alimentar materno e o desenvolvimento de alergias alimentares por parte de seus filhos.

Em relação ao consumo de oleaginosas um estudo mostrou que o consumo materno frequente de amendoim durante a gravidez aumentou o risco de sensibilização entre bebês atópicos de forma dose-dependente (SICHERER et al., 2010) .Outro estudo também encontrou que bebês de mães que consumiram mais amendoim durante a gravidez tiveram 4,22 vezes maior risco de alergia ao mesmo (DESROCHES et al., 2010). Por outro lado duas outras coortes dos Estados Unidos relataram que o consumo materno de amendoim durante a gravidez significativamente reduziu o risco de alergia ao amendoim nos bebês (BUNYAVANICH et al., 2014; FRAZIER et al., 2014). Ainda alguns estudos não encontraram associações entre consumo materno de nozes durante a gravidez e o risco de sensibilização entre as crianças, embora estes estudos de base populacional podem ser insuficientes devido a um pequeno número de crianças com alergia alimentar. (TARIQ et al., 1996; LACK et al., 2003).

Investigou-se também a associação entre a vitamina D sérica da mãe e a associação com o desenvolvimento de alergia alimentar infantil. Um estudo conduzido na Alemanha demonstrou que a concentração de vitamina D no soro de mulheres grávidas e no sangue do cordão umbilical foi positivamente associada ao aumento do risco de desenvolvimento de alergia alimentar entre crianças nos primeiros 2 anos de vida (WEISSE et al., 2013). Um estudo finlandês mostrou que a suplementação de ácido fólico e de vitamina $\mathrm{D}$ durante a gravidez foi associada a um risco aumentado de alergia ao leite de vaca nas crianças dessas mães (TUOKKOLA et al., 2016). Apesar desses dados outros vários estudos não encontraram associação entre os parâmetros bioquímicos maternos de vitamina $\mathrm{D}$ e 
alergia alimentar infantil (JONES et al., 2012; STELMACH et al., 2015), além de um estudo mostrar que níveis mais elevados de vitamina $\mathrm{D}$ materna foram associados com menor alergia alimentar ao amendoim (MULLINS et al., 2012).

Dados de um estudo de coorte trazem que o alto consumo de frutos do mar por parte das mães parece reduzir a sensibilização a alérgenos semelhantes em seus bebês (LOO et al., 2017). Dunstan e colaboradores (2003) relataram risco reduzido de sensibilização para a proteína do ovo, mas não para a do amendoim, nos bebês de mães que suplementaram óleo de peixe durante a gestação. Corroborando com um estudo realizado com mais de 700 mulheres grávidas onde não conseguiram reduzir a alergia alimentar e sensibilização ao amendoim, mas apenas sensibilização ao ovo em bebês de um ano (PALMER et al., 2012).

Os principais consensos e diretrizes (MURARO et al., 2014; SCHUSSLER et al., 2017; SOLÉ et al., 2018) aconselham consistentemente todas as mães terem uma dieta normal e balanceada sem restrições durante a gravidez, uma vez que, restringir alérgenos alimentares para mulheres grávidas parece ter pouco ou nenhum efeito sobre a alergia alimentar na primeira infância, mas as evidências são muito incertas.

\section{ALEITAMENTO MATERNO}

Há algum tempo já foi hipotetizado que as mães podem inadvertidamente sensibilizar seus filhos a certos alimentos através do leite materno (SZAJEWSKA, 2012; GAMBONI; ALLEN; NIXON, 2013), mas atualmente há poucas evidências de que mudar o que as mães consomem durante a amamentação evita alergia alimentar em bebês. Estudos da década de 80 e 90 já mostravam não haver evidências que mudanças da alimentação materna poderia prevenir alergias alimentares em bebês com pré-disposição para tal (HATTEVIG et al., 1989; LOVEGROVE; HAMPTON; MORGAN, 1994). Da mesma forma um estudo com suplementação de probiótico (KALLIOMAKI et al., 2001) e dois estudos com suplementação de óleo de peixe (FURUHJELM et al., 2011; MANLEY et al., 2011) não obtiveram resultados significativos.

Curiosamente ao realizarem uma segunda análise de um estudo de coorte, não foi a restrição, mas sim a introdução de alimentos potencialmente alergênicos que acarretaram em proteção ao desenvolvimento de alergia alimentar nas crianças. Foi constatado que o consumo alimentar de amendoim durante o período de amamentação em combinação com a ingestão precoce desse alimento na infãncia, por volta de 1 ano de idade reduziu significantemente $(\mathrm{p}<0,05)$ a incidência de alergia alimentar (PÌTT et al., 2018).

Os dados sobre a amamentação como estratégia de prevenção da alergia alimentar são contraditórios ou inconclusivos, com diminuição (KULL et al., 2010), aumento (BREW et al., 2012) e falta de associação (VENTER et al., 2009) tendo sido encontrados nos resultados dos estudos. 
Um estudo bastante recente conseguiu demonstrar que para as crianças investigadas a cada mês adicional de amamentação um menor risco de desenvolver alergia alimentar (VAN GINKEL et al., 2018). Entretanto há diversas limitações da literatura sobre os benefícios ou não do aleitamento materno, como: delineamento dos estudos, muitos com durações de amamentação não uniformes e critérios diagnósticos variáveis para alergia alimentar. Apesar disso amamentação exclusiva para os primeiros 4 a 6 meses de idade é recomendado por muitos outros motivos, embora as diretrizes atuais não observam nenhum benefício consistente como meio de prevenção de alergia alimentar (CHAN; CUMMINGS, 2013; SOLÉ et al., 2018).

\section{UTILIZAÇÃO DE FÓRMULAS INFANTIS}

Atualmente os dados em relação a utilização de fórmulas infantis e prevenção de alergias alimentares ainda se mostram heterogêneos. Há ensaios clínicos randomizados com evidências positivas em relação à utilização de fórmulas extensamente hidrolisada (CANANI et al., 2017; NOWAK-WEGRZYN et al., 2019) mas também há estudos que falharam em encontrar esse benefícios (KUO et al., 2011; GOLDSNITH et al., 2016). Algumas diretrizes antigas recomendavam o uso de fórmula hidrolisada para bebês de alto risco quando as mães não podem, ou optaram por não amamentar, como meio de prevenção de alergia alimentar (CHAN; CUMMINGS, 2013).

Entretanto um estudo prospectivo que teve como objetivo determinar os fatores de risco para o desenvolvimento de alergia ao leite de vaca descobriu que bebês que começaram a tomar fórmula à base de proteína do leite de vaca nos primeiros 14 dias de vida tinham taxas mais baixas de alergia ao leite de vaca mediada por IgE em comparação com aqueles em que a fórmula ao leite de vaca foi introduzida entre 105 e 194 dias de vida, 0,05\% contra 1,75\%, respectivamente ( $<0,001)$ (KATZ et al., 2010). Em relação há utilização de fórmulas à base de soja também há muitas incertezas, ensaios clínicos randomizados encontraram que as fórmulas à base de soja podem não proteger contra alergias alimentares em comparação as outras alternativas (LOWE et al., 2011; YAN et al., 2011). Além disso, a introdução da fórmula de soja deve ser realizada somente para crianças maiores de seis meses e que não apresentem manifestações gastrointestinais, não sendo assim uma escolha possível para a prevenção de alergias alimentares antes dessa idade (SOLÉ et al., 2018).

É preciso salientar que as orientações vigentes foram parcialmente baseadas em uma revisão da Cochrane que observou «evidências limitadas» que a fórmula hidrolisada em comparação com a fórmula de leite de vaca regular reduziria o risco de alergia infantil e alergia ao leite de vaca (OSBORN; SINN, 2006). No entanto, análises mais recentes, como uma meta-análise com 37 estudos e mais de 19 mil participantes não observou nenhuma evidência de que fórmulas parcialmente ou extensivamente hidrolisadas reduzissem efetivamente o risco de qualquer doença alérgica, além de apontar para um viés significativo de publicação (BOYLE et al., 2016). Assim as novas diretrizes 
estão começando a questionar e refletir a respeito da real evidência e necessidade da utilização de fórmulas hidrolisadas como meio de prevenção de alergias alimentares (NETTING; CAMPBELL; KOPLIN, 2017). A Academia Americana de Pediatria em seu Comitê de Nutrição coloca que faltam evidências de que fórmulas parcialmente ou extensamente hidrolisadas previnem doenças atópicas em bebês e crianças, mesmo naqueles com alto risco de doença alérgica (GREER et al., 2019). No ano seguinte, em sua nova publicação, reiteram que não há provas demonstrando a eficácia na prevenção de alergia alimentar com o uso da fórmula hidrolisada (COSME-BLANCO; ARROYO-FLORES; ALE, 2020). Do mesmo modo a nova diretriz Canadense traz que não há benefício protetor do uso da fórmula hidrolisada no primeiro ano de vida contra alergia alimentar ou sensibilização alimentar (FLEISCHER et al., 2021).

\section{INTRODUÇÃO ALIMENTAR}

A hipótese de que a exposição a alimentos sólidos na primeira infância poderia aumentar o risco de sensibilização alérgica devido à imaturidade e permeabilidade da barreira da mucosa intestinal levou a as recomendações de que os alimentos alergênicos devem ser evitados durante a introdução alimentar (HOST et al., 1999). Realmente há estudos que encontraram que a introdução alimentar precoce leva a um risco aumentado de alergia alimentar, principalmente quando essa introdução ocorre antes dos 6 meses de vida (KAJOSAARI; SAARINEN, 1983, HOST et al., 1999). Academia Americana de Pediatria (2000) sugeriu postergar a introdução dos laticínios para depois do primeiro ano, do ovo após no segundo ano, e amendoim, nozes e peixes após o terceiro ano de vida para crianças suceptíveias e em 2006, o Colégio Americano de Alergia, Asma e Imunologia estendeu a recomendação para a introdução tardia de potenciais alergênicos para todas as crianças, independentemente do risco de atopia (FIOCCHI; ASSA'AD; BAHNA, 2006).

Entretanto subsequentes estudos observacionais mostraram que atrasar a introdução de potenciais alimentos alergênicos pode na realidade aumentar o risco de sensibilização e alergia alimentar (DU TOIT et al., 2008; ZUTAVERN et al., 2008; KOPLIN et al., 2010; NWARU et al., 2010). Ao mesmo tempo levantaram a hipótese de que a ingestão precoce de pode até ser protetora contra o desenvolvimento de alergia alimentar (DU TOIT et al., 2008; KOPLIN et al., 2010; NWARU et al., 2010). Assim em 2008, a Academia Americana de Pediatria atualizou o recomendações anteriores, reconhecendo que não havia evidências suficientes para apoiar evitar e atrasar a introdução de alérgenos alimentares potenciais na dieta de bebês como um meio para o prevenção primária de alergias alimentares (GREER; SICHERER; BURKS, 2008). Em 2010, pela primeira vez, um estudo transversal de base populacional sugeriu que a introdução de ovos de galinha para dietas infantis entre 4 e 6 meses pode ser uma defesa contra a alergia ao ovo (KOPLIN et al., 2010). Com relação ao cozimento, altas taxas de reação são descritas na introdução de ovo cru 
(PALMER et al., 2013, BELLACH et al., 2017), enquanto ovos cozidos são mais bem tolerados (SICHERER; SAMPSON, 2018, BIRD et al., 2019).

Ainda há estudos recentes que não encontraram associações significativas entre os momento de introdução de comidas e bebidas e risco de desenvolver alergia a alimentos (SANDINI et al., 2011; LUCCIOLI et al., 2014; MCGOWAN et al., 2015). Como é o caso do estudo de Perkin e colaboradores (2016) que analisou o efeito da introdução precoce de trigo e peixe juntamente com outros quatro alimentos alergênicos (amendoim, ovo cozido, leite de vaca e gergelim) e nos resultados deste estudo não houve casos de alergia ao trigo em ambos os grupos, enquanto a taxa de alergia a peixes não foi significativamente diferente no grupo de introdução precoce e no grupo de introdução padrão (após os 6 meses).

Com base nos estudos que temos até o momento parece não haver evidências para adiar a introdução alimentar de alimentos potencialmente alergênicos. No entanto, permanece a recomendação das principais diretrizes e consenso de saúde atuais que orientam o aleitamento materno exclusivo até os 6 meses de vida (WHO, 2003; ASCIA, 2016; THAM et al., 2018) com introdução alimentar progressiva durante o primeiro ano, de acordo com o capacidade de neurológica da criança e hábitos alimentares dos familiares e/ou culturais.

\section{CONCLUSÃO}

A restrição de alimentos durante a gravidez, lactação e introdução alimentar apresenta resultados conflitantes em relação a prevenção das alergias alimentares infantis. Embora seja estabelecido que a ingestão alimentar materna desempenha um papel fundamental no crescimento e desenvolvimento fetal e o aleitamento materno, assim como, a introdução alimentar, afetam a saúde dos indivíduos ao longo de toda sua vida, a forma como isso pode se relacionar com a manifestação de alergias alimentares ainda exige uma investigação mais aprofundada. Mesmo que a importância da dieta e sua relação com alergia alimentar não seja contestada mais estudos são necessários para otimizar as recomendações nutricionais vigentes.

\section{REFERÊNCIAS}

AMERICAN ACADEMY OF PEDIATRICS. Committee on Nutrition. Hypoallergenic infant formulas. Pediatrics, v. 106, n.1, p. 346-349, 2000.

AUSTRALASIAN SOCIETY OF CLINICAL IMMUNOLOGY AND ALLERGY. ASCIA guidelines for infant feeding and allergy prevention. 2016. 
BELLACH, J.; SCHWARZ, V.; AHRENS, B.; TRENDELENBURG, V.; AKSUNGER, Ö.; KALB, B.; NIGGEMANN, B.; KEIL, T.; BEYER, K. Randomized placebo-controlled trial of hen's egg consumption for primary prevention in infants. Journal of Allergy and Clinical Immunology, v. 139, p. 1591-1599, 2017.

BENEDÉ, S.; BLÁZQUEZ, A. B.; CHIANG, D.; TORDESILLAS, L.; BERIN, C. The rise of food allergy: Environmental factors and emerging treatments. The lancet, v. 7, p. 27-34, 2016.

BIRD, J. A.; PARRISH, C.; PATEL, K.; SHIH, J. A.; VICKERY, B.P. Prevention of food allergy: Beyond peanut. Journal of Allergy and Clinical Immunology, v. 143, p. 545-547, 2019.

BJORKSTÉN, B. Genetic and environmental risk factors for the development of food allergy. Current Opinion in Allergy and Clinical Immunology, v. 5, n. 3, p. 249-53, 2005.

BOYCE J. A.; ASSA'A A.; BURKS, A. W.; et al. Guidelines for the diagnosis and management of food allergy in the United States: summary of the NIAID Sponsored Expert Panel Report. Nutrition, v. 27, p. 253-267, 2011.

BOYLE, R. J.; IERODIAKONOU, D.; KHAN, T.; et al. Hydrolysed formula and risk of allergic or autoimmune disease: systematic review and meta-analysis. The BMJ, v. 352, p. i974, 2016.

BREW, B.K.; KULL, I.; GARDEN, F.; ALMQVIST, C.; BERGSTROM, A.; LIND, T.; et al. Breastfeeding, asthma, and allergy: a tale of two cities. Pediatric Allergy and Immunology, v. 23, p. 75-82, 2012.

BUNYAVANICH, S.; RIFAS-SHIMAN, S. L.; PLATTS-MILLS, T. A.; et al. Peanut, milk, and wheat intake during pregnancy is associated with reduced allergy and asthma in children. Journal of Allergy and Clinical Immunology, v. 133, p. 1373-1382, 2014.

CANINI, R. B.; COSRANZO, M.; BEDOGNI, G.; AMOROSO, A.; COSENZA, L.; SCALA, C.; GRANATA, V.; NOCERINO, R. Extensively hydrolyzed casein formula containing Lactobacillus rhamnosus GG reduces the occurrence of other allergic manifestations in children with cow's milk allergy: 3-year randomized controlled trial. Journal of Allergy and Clinical Immunology, v. 139, n. 6, p. 1906-1913, 2017.

CHANG, C.; WU, H.; LU, Q. The Epigenetics of Food Allergy. Advances in Experimental Medicine and Biology, v. 1253, p. 141-152, 2020. 
CHAN, E. S.; CUMMINGS, C. Dietary exposures and allergy prevention in high-risk infants: a joint statement with the Canadian Society of Allergy and Clinical Immunology. Paediatrics and Child Health, v. 18, p. 545-54, 2013.

CHATZI, L.; TORRENT, M.; ROMIEU, I.; GARCIA E. R.; FERRER, C.; VIOQUE, J.; et al. Mediterranean diet in pregnancy is protective for wheeze and atopy in childhood. Thorax, v. 63, p. 507-513, 2008.

CIANFERONI, A.; MURARO, A Food-Induced Anaphylaxis. Immunology and Allergy Clinics of North America, v. 32, n. 1, p. 165-195. 2012.

COSME-BLANCO, W.; ARROYO-FLORES, E.; ALE, H. Food Allergies American Academy of Pediatrics. Pediatrics in Review, 2020.

DESROCHES, A.; INFANTE-RIVARD, C.; PARADIS, L.; et al. Peanut allergy: is maternal transmission of antigens during pregnancy and breastfeeding a risk factor? Journal of Investigational Allergology and Clinical Immunology, v. 20, p. 289-294, 2010.

DUNSTAN, J. A.; MORI, T. A.; BARDEN, A.; BEILIN, L. J.; TAYLOR, A. L.; HOLT, P. G.; et al. Fish oil supplementation in pregnancy modifies neonatal allergen-specific immune responses and clinical outcomes in infants at high risk of atopy: a randomized, controlled trial. Journal of Allergy and Clinical Immunology, v. 112, p. 1178-84, 2003.

DU TOIT, G.; KATZ, Y.; SASIENI, P.; MESHER, D.; MALEKI, S. J.; FISHER, H. R.; FOX, A. T.; TURCANU, V.; AMIR, T.; ZADIK-MNUHUN, G.; et al. Early consumption of peanuts in infancy is associated with a low prevalence of peanut allergy. Journal of Allergy and Clinical Immunology, v. 122, p. 984-991, 2008.

EIWEGGER, T.; HUNG, L.; SAN DIEGO, K.E.; O’MAHONY, L.; UPTON, J. Recent developments and highlights in food allergy. Allergy, v. 74, p. 2355-2367, 2019.

FIOCCHI, A.; ASSA'AD, A.; BAHNA, S. Food allergy and the introduction of solid foods to infants: A consensus document. Adverse Reactions to Foods Committee, American College of Allergy, Asthma and Immunology. Annals of Allergy, Asthma and Immunology, 2006, v. 97, p. 10-20, 2006. 
FLEISCHER, D. M.; CHAN, E. S.; VENTER, C.; SPERGEL, J. M.; ABRAMS, E. M.; STUKUS, D.; et al. A Consensus Approach to the Primary Prevention of Food Allergy Through Nutrition: Guidance from the American Academy of Allergy, Asthma, and Immunology; American College of Allergy, Asthma, and Immunology; and the Canadian Society for Allergy and Clinical Immunology. The Journal of Allergy and Clinical Immunology: In Practice, v. 9, n. 1, p. 22-43, 2021

FRAZIER, A. L.; CAMARGO, C. A.; MALSPEIS, S.; et al. Prospective study of peripregnancy consumption of peanuts or tree nuts by mothers and the risk of peanut or tree nut allergy in their offspring. JAMA Pediatrics, v. 168, p. 156-162, 2014.

FUJIMURA, T.; LUM, S. Z. C.; NAGATA, Y.; KAWAMOTO, S.; OYOSHI, M. K. Influences of Maternal Factors Over Offspring Allergies and the Application for Food Allergy. Frontiers in Immunology, v. 10, p. 1933, 2019.

FURUHJELM, C.; WARSTEDT, K.; FAGERAS, M.; FEALTH-MAGNUSSON, K.; LARSSON, J.; FREDRIKSSON, M.; et al. Allergic disease in infants up to 2 years of age in relation to plasma omega-3 fatty acids and maternal fish oil supplementation in pregnancy and lactation. Pediatric Allergy and Immunology, v. 22, p. 505-514, 2011.

GAMBONI, S. E.; ALLEN, K. J.; NIXON R. L. Infant feeding and the development of food allergies and atopic eczema: an update. Australasian Journal of Dermatology, v. 54, p. 85-89, 2013.

GOLDSMITH, A. J.; KOPLIN, J. J.; LOWE, A. J.; TANG, M. L.; MATHESON, M. C.; ROBINSON, M.; PETERS, R., DHARMAGE, S. C.; ALLEN, K. J. Formula and breast feeding in infant food allergy: A population-based study. Journal of Paediatrics and Child Health, v. 52, n. 4, p. 377-84, 2016.

GREER, F. R.; SICHERER, S. H.; BURKS, A. W. Effects of early nutritional interventions on the development of atopic disease in infants and children: The role of maternal dietary restriction, breastfeeding, timing of introduction of complementary foods, and hydrolyzed formulas. Pediatrics, v. 121, p. 183-191, 2008.

GREER, F. R.; SICHERER, S. H.; BURKS, A. W.; AAP COMMITTEE ON NUTRITION; AAP SECTION ON ALLERGY AND IMMUNOLOGY. The Effects of Early Nutritional Interventions on the Development of Atopic Disease in Infants and Children: The Role of Maternal Dietary Restriction, Breastfeeding, Hydrolyzed Formulas, and Timing of Introduction of Allergenic Complementary Foods. Pediatrics, v. 143, n. 4, p. e20190281, 2019 
GUPTA R. S.; WARREN C. M.; SMITH, B. M.; JIANG, J.; BLUMENSTOCK, J. A.; DAVIS, M. M.; et al. Prevalence and severity of food allergies among US adults. JAMA Network Open, p. e185630, 2019.

HALFON, N.; LARSON, K.; LU, M.; TULLIS, E.; RUSS, S. Lifecourse health development: past, present and future. Maternal and Child Health Journal, v. 18, n. 2, p. 344-65, 2014.

HATTEVIG, G.; KJELLMAN, B.; SIGURS, N.; BJORKST, B.; KJELLMAN, N. I. Effect of maternal avoidance of eggs, cow's milk and fish during lactation upon allergic manifestations in infants. Clinical and Experimental Allergy, v. 19, p. 27-32, 1989.

HOST, A.; KOLETZKO, B.; DREBORG, S.; MURARO, A.; WAHN, U.; AGGETT, P.; BRESSON, J. L.; HERNELL, O.; LAFEBER, H.; MICHELSEN, K.F.; et al. Dietary products used in infants for treatment and prevention of food allergy. Joint Statement of the European Society for Paediatric Allergology and Clinical Immunology (ESPACI) Committee on Hypoallergenic Formulas and the European Society for Paediatric Gastroenterology, Hepatology and Nutrition (ESPGHAN) Committee on Nutrition. Archives of Disease in Childhood, v. 81, p. 80-84, 1999.

JONES, A. P.; PALMER, D.; ZHANG, G.; et al. Cord blood 25-hydroxyvitamin D3 and allergic disease during infancy. Pediatrics, 2012;130:e1128-e1135.

KAJOSAARI, M.; SAARINEN, U. M. Prophylaxis of atopic disease by six months' total solid food elimination. Evaluation of 135 exclusively breast-fed infants of atopic families. Acta Paediatrica Scandinavica, v. 72, p. 411-4, 1983.

KALLIOMAKI, M.; SALMINEN, S.; ARVILOMMI, H.; KERO, P.; KOSKINEN, P.; ISOLAURI, E. Probiotics in primary prevention of atopic disease: a randomised placebo-controlled trial. Lancet, v. 357, p. 1076-1079, 2001.

KATZ, Y.; RAJUAN, N.; GOLDBERG, M. R.; EISENBERG, E.; HEYMAN, E.; COHEN, A.; et al. Early exposure to cow's milk protein is protective against IgE-mediated cow's milk protein allergy. Journal of Allergy and Clinical Immunology, v. 126, p. 77-82, 2010.

KEMP, A. S.; PONSONBY, A. L.; DWYER, T.; COCHRANE, J. A.; PEZIC, A.; JONES, G. Maternal antenatal peanut consumption and peanut and rye sensitization in the offspring at adolescence. Clinical and Experimental Allergy, v. 41, p. 224-231, 2011. 
KOPLIN, J. J.; OSBORNE, N. J.; WAKE, M.; MARTIN, P. E.; GURRIN, L. C.; ROBINSON, M. N.; TEY, D.; SLAA, M.; THIELE, L.; MILES, L.; et al. Can early introduction of egg prevent egg allergy in infants? A population-based study. Journal of Allergy and Clinical Immunology, v. 126, p. 807-813, 2010.

KUlL, I.; MELEN, E.; ALM, J.; HALlBERG, J.; SYARTENGREN, M.; VAN HAGE, M.; et al. Breast-feeding in relation to asthma, lung function, and sensitization in young school children. Journal of Allergy and Clinical Immunology, v. 125, p. 1013-9, 2010.

KUO, H. C.; LIU, C. A.; OU, C. Y.; HSU, T. Y.; WANG, C. L.; HUANG, H. C.; CHUANG, H.; LIANG, H. M.; YANG, K. D. Partial protein-hydrolyzed infant formula decreased food sensitization but not allergic disease in a prospective birth cohort study. International Archives of Allergy and Immunology, v. 154, p. 310- 317, 2011.

LACK, G.; FOX, D.; NORTHSTONE, K.; GOLDING, J. Avon Longitudinal Study of Parents and Children Study Team. Factors associated with the development of peanut allergy in childhood. The New England Journal of Medicine, v. 348, p. 977-85, 2003.

LOO, E. X. L.; ONG, L.; GOH, A.; et al. Effect of maternal dietary patterns during pregnancy on self-reported allergic diseases in the first 3 years of life: results from the GUSTO study.International Archives of Allergy and Immunology, v. 173, p. 105-113, 2017.

LOVEGROVE, J. A.; HAMPTON, S. M.; MORGAN, J. B. The immunological and long-term atopic outcome of infants born to women following a milk-free diet during late pregnancy and lactation: a pilot study. British Journal of Nutrition, v. 71, p. 223-238, 1994.

LOWE, A. J.; HOSKING, C. S.; BENNETT, C. M.; ALLEN, K. J.; AXELRAD, C.; CARLIN, J. B.; et al. Effect of a partially hydrolyzed whey infant formula at weaning on risk of allergic disease in highrisk children: a randomized controlled trial. Journal of Allergy and Clinical Immunology, v. 128 , p. $360-365,2011$.

LUCCIOLI, S.; ZHANG, Y.; VERRILL, L.; RAMOS-VALLE M.; KWEGYIR-AFFUL E. Infant feeding practices and reported food allergies at 6 years of age. Pediatrics, v. 134, n. 1, p. S21-8, 2014.

MANLEY, B. J.; MAKRIDES, M.; COLLINS, C. T.; MCPHEE, A. J.; GIBSON, R. A.; RYAN, P.; et al. High-dose docosahexaenoic acid supplementation of preterm infants: respiratory and allergy outcomes. Pediatrics, v. 128, p. e71-e77, 2011. 
MCGOWAN E. C.; BLOOMBERG, G. R.; GERGEN, P. J.; VISNESS, C. M.; JAFFEE, K. F.; SANDEL, M.; O’CONNOR, G.; KATTAN, M.; GERN, J.; WOOD, R. A. Influence of early life exposures on food sensitization and food allergy in an inner-city birth cohort. Journal of Allergy and Clinical Immunology, v. 135, p. 171-8, 2015.

MULLINS, R. J.; CLARK, S.; WILEY, V.; et al. Neonatal vitamin D status and childhood peanut allergy: a pilot study. Annals of Allergy, Asthma and Immunology, v. 109, p. 324-328, 2012.

MURARO, A.; HALKEN, S.; ARSHAD, S. H.; et al. EAACI food allergy and anaphylaxis guidelines. Primary prevention of food allergy. Allergy, v. 69, p. 590-601, 2014.

MURARO, A.; HERNANDEZ, D. A. M. Managing food allergy and anaphylaxis: A new model for an integrated approach. Allergology International, v. 69, n. 1, p. 19-27, 2020.

NETTING, M. J.; CAMPBELL, D. E.; KOPLIN, J. J. An Australian Consensus on infant feeding guidelines to prevent food allergy: outcomes from the Australian infant feeding Summit. The Journal of Allergy and Clinical Immunology: In Practice, v. 5, p. 1617-24, 2017.

NOWAK-WEGRZYN, A.; CZERKIES, L.; REYES, K.; COLLINS, B.; HEINE, R. G. Confirmed Hypoallergenicity of a Novel Whey-Based Extensively Hydrolyzed Infant Formula Containing Two Human Milk Oligosaccharides. Nutrientes, v. 11, n. 7, p. 1447, 2019.

NWARU, B. I.; ERKKOLA, M.; AHONEN, S.; KAILA, M.; HAAPALA, A. M.; KRONBERGKIPPILA, C.; SALMELIN, R.; VEIJOLA, R.; ILONEN, J.; SIMELL, O.; et al. Age at the introduction of solid foods during the first year and allergic sensitization at age 5 years. Pediatrics, v. 125, p. 50-59, 2010.

OSBORN, D. A.; SINN, J. Formulas containing hydrolysed protein for prevention of allergy and food intolerance in infants. Cochrane Database of Systematic Reviews, v. 4, 2006.

PALMER, D. J.; METCALFE, J.; MAKRIDES, M.; GOLD, M. S.; QUINN, P.; WEST, C. E.; LOH, R.; PRESCOTT, S. L. Early regular egg exposure in infants with eczema: A randomized controlled trial. Journal of Allergy and Clinical Immunology, v. 132, p. 387-392, 2013.

PALMER, D. J.; SULLIVAN, T.; GOLD, M. S.; PRESCOOT, S. L.; HEDDLE, R.; GIBSON, R. A.; et al. Effect of n-3 long chain polyunsaturated fatty acid supplementation in pregnancy on infants' allergies in first year of life: randomised controlled trial. The BMJ, p. 344:e184, 2012. 
PERKIN, M. R.; LOGAN, K.; TSENG, A.; RAJI, B.; AYIS, S.; PEACOCK, J.; BROUGH, H.; MARRS, T.; RADULOVIC, S.; CRAVEN, J.; et al. Randomized Trial of Introduction of Allergenic Foods in Breast-Fed Infants. The New England Journal of Medicine, v. 374, p. 1733-174, 2016.

PITT, T. J.; BECKER, A. B.; CHAN-YEUNG, M.; et al. Reduced risk of peanut sensitization following exposure through breast-feeding and early peanut introduction. Journal of Allergy and Clinical Immunology, v.141, p. 620-5, 2018.

SANDINI, U.; KUKKONEN, A. K.; POUSSA, T.; SANDINI, L.; SAVILAHTI, E.; KUITUNEN, M. Protective and risk factors for allergic diseases in high-risk children at the ages of two and five years. International Archives of Allergy and Immunology, v. 156, n. 3, p. 339-48, 2011.

SCHUSSLER, E.; SOBEL, J.; HSU, J.; YU, P.; MEANEY-DELMAN, D.; GRAMMER, L.C.; NOWAL-WEGRZYN, A.Workgroup Report by the Joint Task Force Involving American Academy of Allergy, Asthma \& Immunology (AAAAI); Food Allergy, Anaphylaxis, Dermatology and Drug Allergy (FADDA) (Adverse Reactions to Foods Committee and Adverse Reactions to Drugs, Biologicals, and Latex Committee); and the Centers for Disease Control and Prevention Botulism Clinical Treatment Guidelines Work group-Allergic Reactions to Botulinum Antitoxin: A Systematic Review. Clinical Infectious Diseases, v. 66, p. S65-S72, 2017.

SICHERER, S. H.; SAMPSON, H. A. Food allergy: A review and update on epidemiology, pathogenesis, diagnosis, prevention, and management. Journal of Allergy and Clinical Immunology, v. 141, n. 1, p. 41-58, 2018.

SICHERER S. H.; WOOD, R. A.; STABLEIN, D.; LINDBLAD, R.; BURKS, A. W.; LIU, A. H.; et al. Maternal consumption of peanut during pregnancy is associated with peanut sensitization in atopic infants. Journal of Allergy and Clinical Immunology, v. 126, p. 1191-7, 2010.

SOLÉ, D.;SILVA L. R.; COCCO R. R.; FERREIRA, C. T.; SARNI, R. O.; OLIVEIRA, L. C.; et al. Brazilian Consensus on Food Allergy: 2018 - Part 2 - Diagnosis, treatment and prevention. Joint position paper of the Brazilian Society of Pediatrics and the Brazilian Association of Allergy and Immunology. Arquivis de Asma, Alergia e Imunologia, v. 2, n. 1, 2018.

STELMACH, I.; MAJAK, P.; JERZYNSKA, J.; et al. Cord serum 25-hydroxyvitamin D correlates with early childhood viral-induced wheezing. Respiratory Medicine, v. 109, p. 38-43, 2015. 
SZAJEWSKA, H. Early nutritional strategies for preventing allergic disease. The Israel Medical Association Journal, v. 14, p. 58-62, 2012.

TANG, M. L. K.; MULLINS, R. J. Food allergy: is prevalence increasing? International Medicine Journal, v. 47, n. 3, p. 256-261, 2017.

TARIQ, S. M.; STEVENS, M.; MATTHEWS, S.; RIDOUT S.; TWISELTON, R.; HIDE, D. W. Cohort study of peanut and tree nut sensitisation by age of 4 years. THE BMJ, v. 313, p. 514-7, 1996.

THAM, E. H.; SHEK, L. P.; VAN BEVER, H. P.; VICHYANOND, P.; EBISAWA, M.; WONG, G. W.; LEE, B. W.; Asia Pacific Association of Pediatric Allergy, Respirology \& Immunology (APAPARI). Early introduction of allergenic foods for the prevention of food allergy from an Asian perspectiveAn Asia Pacific Association of Pediatric Allergy, Respirology \& Immunology (APAPARI) consensus statement. Pediatric Allergy and Immunology, v. 29, 18-27, 2018.

TUOKKOLA, J.; LUUKKAINEN, P.; KAILA, M.; et al. Maternal dietary folate, folic acid and vitamin D intakes during pregnancy and lactation and the risk of cows' milk allergy in the offspring. British Journal of Nutrition, v. 116, p. 710-718, 2016.

UNTERSMAYR, E.; JENSEN-JAROLIM, E. The role of protein digestibility and antacids on food allergy outcomes. Journal of Allergy and Clinical Immunology, v. 121, n. 6, p. 1301-1310, 2008.

VALENTA, R.; HOCHWALLNER, H.; LINHART, B.; PAHR, S. Food Allergies: The Basics. Gastroenterology, v. 148, p.1120-1131, 2015.

VAN GINKEL, C. D.; VAN DER MEULEN, G. N.; BAK, E.; FLOKSTRA-DE BLOK B. M. J.; KOLLEN, B. J.; KOPPELMAN, G. H.; DUBOIS, A. E. J. Retrospective observational cohort study regarding the effect of breastfeeding on challenge-proven food allergy. European Journal of Clinical Nutrition, v. 72, n. 4, p. 557-563, 2018.

VENTER, C.; PEREIRA, B.; VOIGT, K.; GRUNDY, J.; CLAYTON, C. B.; HIGGINS, B.; et al. Factors associated with maternal dietary intake, feeding and weaning practices, and the development of food hypersensitivity in the infant. Pediatric Allergy and Immunology, v. 20, p. 320-7, 2009. 
WEISSE, K.; WINKLER, S.; HIRCHE, F.; HERBERTH, G.; HINZ, D.; BAUER, M.; et al. Maternal and newborn vitamin D status and its impact on food allergy development in the German LINA cohort study. Allergy, v. 68, p. 220-8, 2013.

WHO. Global Strategy for Infant and Young Child Feeding; World Health Organization: Geneva, Switzerland, 2003.

YAN, J.; CHEN, J.; LI, H.; HU, Y. Evaluation of therapeutic effects of three hypoallergenic formulae in infants with cow's milk protein allergy. Chinese Journal of Pediatrics, v. 49, n. 7, p. 501-5, 2011.

ZUTAVERN, A.; BROCKOW, I.; SCHAAF, B.; VON BERG, A.; DIEZ, U.; BORTE, M.; KRAEMER, U.; HERBARTH, O.; BEHRENDT, H.; WICHMANN, H.E.; et al. Timing of solid food introduction in relation to eczema, asthma, allergic rhinitis, and food and inhalant sensitization at the age of 6 years: Results from the prospective birth cohort study LISA. Pediatrics, v. 121, p. e44-e52, 2008. 